\title{
Le peculiarità altoatesine nella narrativa italiana: il caso di Eva dorme di Francesca Melandri
}

\section{Abstract \\ The South Tyrol's peculiarities in contemporary italian literature: the case of Eva dorme by Francesca Melandri}

The article deals with the subject of the South Tyrol's peculiarities in Francesca Melandri's debut novel Eva dorme (Eve sleeps, 2011). The author presents the most important characteristics of the region - the multilingualism of province, lack of understanding between the different ethnic groups, relationships with newcomers, reciprocal stereotypes and the legal separation of italian- and german-speaking groups. The novel is an interesting and original position on italian publishing market (due to a subject of novel) but it represents a conservative attempt to the problem of local identity and didactics aspiration to approach a less well-known part of country to the italian reader.

Key words: South Tyrol, contemporary italian literature, regional identity, Francesca Melandri

Parole chiave: Alto Adige, Sudtirolo, letteratura italiana contemporanea, identità regionale, Francesca Melandri 
L'Alto Adige è una delle province meno conosciute d'Italia. Un piccolo microcosmo situato all'estremo lato settentrionale dello Stato che non è molto presente nella riflessione letteraria o culturale riguardante l'Appennino. Una delle prove più note che cerca di colmare questo vuoto nell'ambito editoriale e letterario italiano degli ultimi anni è il romanzo Eva dorme di Francesca Melandri. II libro pubblicato da Mondadori nel 2011 ha avuto un grande successo in Italia. Peraltro è il primo romanzo sulla tematica altoatesina indirizzato ad un pubblico così vasto, in grado di oltrepassare il cerchio letterario locale.

Eva dorme è un romanzo che tratta della storia altoatesina attraverso il racconto della vita di due donne: Gerda e Eva, madre e figlia, immerse nella complicata specificità locale. La questione regionale non è l'unico tema del libro, ma è quello su cui ci soffermeremo nel presente articolo.

\section{Geografia, storia e politica dell'Alto Adige}

Prima di cominciare la riflessione sugli aspetti letterari è opportuno riportare qualche informazione generale: cos'è esattamente l'Alto Adige, dove si trova, qual era la sua storia, com'è il suo status politico attuale e in che modo esso influisce sulla vita quotidiana.

Il nome 'Alto Adige' è quello più utilizzato per denominare la terra di cui si tratta nel presente testo. Tuttavia il suo nome ufficiale è La Provincia Autonoma di Bolzano-Alto Adige (chiamata in tedesco Autonome Provinz Bozen-Südtirol e in ladino Provinzia Autonoma de Balsan/Bulsan) e insieme con La Provincia Autonoma di Trento (nota come Trentino) costituiscono la regione denominata Trentino-Alto Adige/Südtirol. ${ }^{1}$ Le province sono situate nell'Italia settentrionale, a sud del passo del Brennero (Blanco 2006: 121).

\section{La storia}

Trattando (anche se soltanto in modo sommario) un tema così complesso come la storia della regione, bisogna ricordare che la storiografia dell'Alto Adige/ Südtirol viene spesso usata per diversi scopi ideologici. Per questo motivo, come ammette Luigi Blanco, non esiste una vera e propria storiografia regionale. Quella

1 Le due province sono le uniche due ad essere autonome in Italia. Invece altre aree amministrative a statuto speciale sono sempre le regioni, quattro in Italia: Sicilia, Sardegna, Valle d'Aosta e Friuli-Venezia Giulia.

188

Fabrica Litterarum Polono-Italica | 2019, nr 1 (1) 
esistente è la storiografia utilizzata dai gruppi linguistici per sostenere, difendere e rafforzare il proprio profilo identitario (Blanco 2006: 139). II presente paragrafo ha come unico obiettivo quello di indicare nel modo più oggettivo possibile i momenti più significativi della storia regionale, indispensabili per capire la seguente analisi letteraria, senza però entrare troppo nei dettagli.

Fino alla metà del XIV secolo l'Alto Adige è parte integrante della sovrana contea del Tirolo. Nel 1363 quest'ultima viene ceduta da Margareta Maultasch a Rodolfo IV d'Asburgo e così entra a far parte dell'Impero Asburgico (Hempel 2008: 9), fino all'inizio del XX secolo (quasi ininterrottamente). II territorio dell'attuale Alto Adige è concesso all'Italia in cambio dell'entrata in guerra, in seguito al trattato segreto stipulato nel 1915. Dopo la fine della prima guerra mondiale, nel 1919, il «Paese di cultura alpina e di lingua tedesca [...] viene diviso in due parti in seguito al trattato di Saint-Germain[-en-Laye]» (Vassalli 2015: 24) che conferma l'annessione dell'Alto Adige all'Italia.

Subito dopo l'annessione all'Italia, comincia il periodo di intensa snazionalizzazione e italianizzazione contro la popolazione tirolese e ladina. A capo di tale processo di obbligatoria assimilazione culturale e linguistica delle minoranze nelle ex-terre dell'Impero Asburgico (Sternalski 2013: 51) c'è Ettore Tolomei: "il profeta e l'ideologo del fascismo tra il Brennero e Trento" (Vassalli 2015: 20), definito da Vassalli, come il personaggio "al quale si deve I'“invenzione" dell'Alto Adige» (Blanco 2006: 131). Dal 1918 è convocato a dirigere il Commissariato alla Lingua e alla Cultura per I'Alto Adige a Bolzano; successivamente nel 1923 proclama il manifesto nazionalista intitolato Provvedimenti per l'Alto Adige in cui formula i principi di italianizzazione, tra i quali il divieto dell'uso del nome 'Tirol' sostituito dal nome 'Alto Adige' (Sternalski 2013: 53). Altri provvedimenti che portano alla snazionalizzazione del Tirolo del Sud erano:

- italianizzazione della toponomastica e dei nomi e cognomi tedeschi;

- divieto di insegnamento in tedesco; la sola lingua ammessa a scuola era l'italiano (tale situazione ha provocato la nascita di Katakombenschulen, le scuole clandestine esistite fino al 1943);

- inclusione delle terre del Tirolo del Sud nella provincia di maggioranza italiana del Trento;

- ampliamento delle linee ferroviarie che collegavano I'Italia (Sternalski 2013: 53-54).

Nel 1939, in virtù dell'accordo tra Italia e Germania, viene introdotto il sistema chiamato Opzioni (Blanco 2006: 132) che impone ai sudtirolesi e ai ladini un'alternativa dolorosa. Possono scegliere tra la cittadinanza tedesca, la quale implica il trasferimento obbligatorio nella Germania nazista, oppure quella italiana che avrebbe permesso loro di rimanere nella propria terra, rinunciando così ad essere riconosciuti come una minoranza e imponendo loro l'obbligo di piena italianizza- 
zione. Di conseguenza coloro che optano per la Germania vengono chiamati nazisti, invece quelli che decidono di rimanere in Italia sono accusati di tradimento. Questa situazione difficile, quasi drammatica, è riportata nel romanzo di Francesca Melandri:

[...] l'alternativa non veniva posta tra il restare o il partire, bensì tra il dichiararsi Walsch oppure Daitsch: italiani o tedeschi. Non si poteva restare tedeschi su suolo italiano [...]. Restare [...] era inequivocabile segno di tradimento, insubordinazione contro la causa nazionalsocialista, viltà (Melandri 2017: 32).

Secondo le stime l'80-90\% degli autoctoni si dichiara favorevole all'emigrazione. Tuttavia le Opzioni vengono interrotte dallo scoppio della guerra (Sternalski 2013: 57).

Nel 1943, con la capitolazione dell'Italia, I'Alto Adige viene annesso al Terzo Reich; ciò permette ai tirolesi emigrati, dopo aver scelto l'opzione tedesca, di tornare nella propria terra e agli altri impedisce la partenza l'opzione prescelta per le ragioni politiche, ma emotivamente non voluta. Bisogna ricordare che da una parte tale situazione è per i sudtirolesi fino ad allora non partiti la liberazione dal fascismo, dall'altra però significa immersione nel nazismo. Inoltre, coloro che si sono trasferiti in Germania, ritornando a casa, si ritrovano le proprie abitazioni occupate (dagli italiani).

Dopo la seconda guerra mondiale, si ripresenta il problema riguardante il Paese a cui sarebbe dovuto appartenere l'Alto Adige. L'accordo De Gasperi-Gruber (che prende il nome dal ministro italiano Alcide De Gasperi e dal ministro austriaco Karl Gruber, stipulato a Parigi il 5 settembre 1946) annette la regione al territorio italiano, seppur obbligando gli italiani ad assicurare i diritti degli abitanti germanofoni (Marcantoni, Postal 2014: 20), l'accordo «costituirà la base dell'autonomia sudtirolese e dei suoi successivi sviluppi» (Blanco 2006: 133).

Il primo statuto di autonomia del 1948, valido per entrambe le provincie, non è stato pienamente rispettato. Ciò dà vita a delle proteste, tra le quali la più grande e più importante ha luogo a Castel Firmiano il 17 novembre 1957 guidata da Silvius Magnago, con il motto: Los von Trient! [Via da Trento!]. I partecipanti richiedono «un'autonomia provinciale non legata a un'altra provincia di lingua italiana, come quella di Trento» (Melandri 2017: 53) e i più radicali addirittura un'autonomia da Roma (Los von Rom! [Via da Roma!]) (Melandri 2017: 53).

Nel 1956 viene fondato il BAS (Befreiungsausschuss Sudtirol, letteralmente: Comitato per la liberazione del Sudtirolo) "con l'obiettivo iniziale di mettere in atto una serie di azioni dimostrative per richiamare l'attenzione sul Sudtirolo, sia internamente sia sul piano internazionale» (Marcantoni, Postal 2014: 37). Con esso inizia la prima fase del terrorismo altoatesino, il cui culmine si raggiunge con la 
notte dei fuochi dell'11 giugno 1961. L'obiettivo dell'attentato è quello di far saltare in aria i tralicci e di danneggiare le stazioni ferroviarie, in questa occasione accidentalmente vi muore una persona (Marcantoni, Postal 2014: 47-49).

Tale atto terroristico aggrava maggiormente la situazione già vacillante. Gli italiani temendo un'ulteriore destabilizzazione politica, militarizzano la regione. In conseguenza l'insoddisfazione dei sudtirolesi aumenta, provocando ulteriori atti di violenza. II governo italiano comincia una nuova politica caratterizzata da rappresaglie e retate (Marcantoni, Postal 2014: 49-55).

Durante l'autunno del 1961 cominciano i lavori per il nuovo Statuto di Autonomia. Il disegno del documento chiamato "Pacchetto», scritto da Silvius Magnago, viene approvato seppur con maggioranza minima. La nuova legge entra in vigore nel 1972 e la situazione nella regione finalmente si placa. II secondo Statuto di Autonomia garantisce un'ampia autonomia della provincia (Golemo 2013: 106-110). Le regole più significative introdotte dallo Statuto sono: I'autonomia trattata separatamente per ognuna delle province: di Bolzano e di Trento (Blanco 2006: 134); le competenze importanti trasferite all'ente provinciale con possibilità di ampliamento delle autonomie (Blanco 2006: 134) e soprattutto «la norma più importante, ma anche controversa [...] quella della "proporzionale etnica" in provincia di Bolzano: essa sancisce il criterio di ripartizione dei posti nel pubblico impiego sulla base della consistenza dei gruppi linguistici provinciali, consistenza numerica desunta dai periodici censimenti della popolazione che prevedono anche una dichiarazione di appartenenza» (Blanco 2006: 134-135).

Nel 2001 un'ulteriore riforma della Costituzione Italiana riguardo l'Alto Adige riporta le seguenti modifiche:

- la Regione composta da due province autonome, elementi portanti dell'autonomia territoriale (Blanco 2006: 135);

- il rafforzamento della presenza dei ladini in Consiglio regionale (Blanco 2006: 136);

- l'introduzione ufficiale della denominazione Südtirol (Blanco 2006: 136).

\section{La Proporzionale etnica}

Prima di tutto riguardo la legge della Proporzionale etnica c'è un problema rilevante a livello di definizione. Nell'ambito della ricerca contemporanea non esiste una definizione chiara e univoca del termine 'etnicità'. La legge, invece, ha bisogno di soluzioni trasparenti e precise. II criterio più importante, per non dire unico, per rendere la norma applicabile, è la differenza linguistica. Ogni abitante dell'Alto Adige di 14 anni compiuti, deve dichiarare la propria etnia (in base alla lingua parlata) nel censimento e la sua dichiarazione sarà il fondamento per l'esercizio

191

Fabrica Litterarum Polono-Italica | 2019, nr 1 (1) 
delle norme derivanti dalla proporzionale. Per i minori di 14 anni sono i genitori a dichiararlo. Si può scegliere solamente l'appartenenza al gruppo tedesco, italiano o ladino. Non esiste la possibilità di definirsi «multietnico» o di una quarta etnia. L'unica eccezione alla regola sono i matrimoni misti. La legge prevede la possibilità di non mettersi d'accordo da parte dei genitori sull'identità etnica dei loro figli. In questo unico caso i genitori sono esonerati da dare una dichiarazione per i propri bambini (Kleinert 2012: 66). In una regione da secoli situata al confine, dove si incontrarono tante influenze culturali, può sorprendere che uno debba autodefinirsi in modo così rigido (Kleinert 2012: 66).

Come sostiene Kleinert, nella Proporzionale etnica c'è una sottile insensatezza. Da una parte il censimento non prevede la possibilità di scegliere più di un'appartenenza linguistica. Risulta allora che non esista la possibilità di bilinguismo. Dall'altra, per ottenere qualsiasi lavoro nel settore pubblico bisogna sostenere l'esame di bilinguismo, chiamato «il patentino» (Kleinert 2012: 67).

La Proporzionale e i censimenti tendono alla piena separazione delle popolazioni. La politica provinciale prevede una coesistenza di esse, ma non una convivenza tra loro (Blanco 2006: 135). Tutto ciò provoca una forte e rigida separazione tra i gruppi linguistici. Anche la cultura, in gran parte, è separata. Ci sono addirittura diversi assessorati alla cultura (italiano e tedesco) che raramente comunicano tra di loro e altrettanto sporadicamente organizzano eventi insieme (Blanco 2006: 137). Inoltre ci sono diversi sistemi scolastici e formativi per ogni gruppo (Blanco 2006: 137). Altri casi esemplari della separazione sono le due sezioni della Caritas (italiana ed austriaca) e le due organizzazioni alpine: il Club Alpino Italiano e l'Alpenverein Sudtirol (che hanno persino infrastrutture alpine separate) (Golemo 2013: 120).

\section{Le caratteristiche regionali nel romanzo Eva dorme di Francesca Melandri}

Le condizioni etniche, geopolitiche e culturali, presenti in provincia e distinte da quelle in altre regioni italiane, fanno sì che si crei un tipo d'identità e una cultura locale diverse da quelle predominanti nel resto della Penisola. Le differenze nella comprensione della propria identità tra gli abitanti dell'Alto Adige e delle altre province italiane si possono notare nei diversi aspetti della vita quotidiana e i loro riflessi sono anche visibili nella letteratura. Tra gli scrittori di madrelingua italiana queste differenze sono colte e trasmesse bene da Francesca Melandri nella sua opera Eva dorme. Le tematiche trattate, i problemi e la peculiarità altoatesini che 
costituiscono le componenti dell'identità regionale dei protagonisti e stimolano il suo sviluppo, sono spunto di riflessione per l'autrice.

\section{La lingua}

Indubbiamente, tra i temi trattati nel romanzo, il più rilevante, è la questione della lingua. II bilinguismo, la scarsa padronanza dell'italiano da parte dei sudtirolesi tedescofoni (e del tedesco da parte degli italofoni), il sentimento di vergogna per il parlare male e persino le influenze reciproche tra tedesco e italiano (a volte sorprendenti!) che risultano accomunati, sono tematiche ricorrenti nel testo.

Come già menzionato, il bilinguismo nella regione è attualmente stabilito dalla legge. Vista la forte presenza dei due gruppi linguistici (e un terzo, quello ladino, più limitato territorialmente) il bilinguismo non è solo regolato dal punto di vista giuridico, obbligando le istituzioni pubbliche ad adottare le due lingue, ma è soprattutto una necessità della gente comune. Tale situazione la ritroviamo sin dalle prime pagine del romanzo:

Dopo Sterzig/Vipiteno, poco prima di Franzensfeste/Fortezza, Carlo ha fermato all'Autobahnraststätte/Autogrill e ci siamo mangiati un belegtes Brötche/panino. Poi siamo usciti dall'Autobahn/autostrada e abbiamo pagato al Mautstelle/ casello. Sulla sua Volvo, che meno male è svedese e quindi non si traduce né in tedesco né in italiano. Benvenuti in Südtirol/Alto Adige, regione del bilinguismo (Melandri 2017: 18).

In questo frammento non c'è ancora nessuna traccia emotiva. II bilinguismo è soltanto una coesistenza di più termini in entrambe le lingue utilizzati per denominare la stessa cosa. Nel corso del libro, e della spiegazione al lettore che in Alto Adige niente è così come lo si aspettava, si evincono esempi di una coesistenza linguistica più significativa.

In una regione come l'Alto Adige anche il modo di chiamare il cibo non è neutrale. Con una buona dose d'ironia l'autrice fa vedere come il bilinguismo culinario in realtà rappresenti la mescolanza delle lingue e gli influssi reciproci in tutti gli aspetti della vita. Un esempio di ciò è presentato dalla Melandri, che ci spiega come nei primi anni Sessanta, Herr Neumann, cuoco dell'albergo in cui lavorava la protagonista Gerda, si esprimeva parlando del cibo:

[i pesci] li chiamava così, in italiano. Anche per verdura e frutta usava i nomi italiani [...]. Per la carne, invece, usava il tedesco [...]. Quel bilinguismo culinario [...]. L'unica eccezione alla regola, quasi un omaggio involontario agli stereotipi tra italiani e tedeschi, erano le patate: pur rientrando nella categoria

193

Fabrica Litterarum Polono-Italica | 2019, nr 1 (1) 
degli ortaggi, o almeno dei tuberi, per tutti erano sempre e solo Kartoffeln. Che però come la frittura superavano le tensioni interetniche sudtirolesi e acquisivano status internazionale, diventando Pommes Frites (Melandri 2017: 82).

Pur esistendo la piena corrispondenza tra i due codici lessicali (ogni alimento ha un suo equivalente semantico nell'altra lingua) si è stabilita una suddivisione dei termini, difficile da cogliere in modo logico (i parlanti mescolano le parole provenienti dalle due lingue, usando per alcuni alimenti sempre i nomi tedeschi e per gli altri invece i nomi italiani). Gli abitanti non parlano più né le proprie lingue letterarie né i dialetti nelle loro forme tradizionali.

Un altro ambito in cui è visibile quel intrecciarsi delle lingue è quello delle imprecazioni. Con la sua solita ironia l'autrice, parlando di un personaggio, descrive così la situazione:

Era Daitsch ${ }^{2}$, ma bestemmiava in italiano. Tutti i sudtirolesi ora bestemmiavano in italiano [...]. L'adozione unanime della bestemmia italiana da parte della popolazione di lingua tedesca fu, dell'italianizzazione forzata voluta dal fascismo, l'unico ma, bisogna dire, imperituro successo (Melandri 2017: 54).

Si potrebbe chiedere, perché proprio le espressioni ingiuriose furono adottate con più facilità? Magari è una domanda superflua, vista l'esperienza di tante persone che, nell'imparare una lingua straniera acquisiscono in primo luogo le parolacce e le bestemmie. Può darsi però che la ragione sia più profonda e più significativa. L'uso delle imprecazioni, nel frammento su citato, è associato all'italianizzazione ai tempi del fascismo. Ovvero un evento forzato, che -si può sospettare -procurò nei sudtirolesi rabbia e frustrazione. I fascisti nel Ventennio costringevano la popolazione sudtirolese a rispettare le istituzioni statali e a parlare solo italiano. Di conseguenza, l'adozione delle bestemmie può essere vista come un sottile cinismo, se non addirittura come una cripto-insurrezione. Non potendo opporsi alle leggi fasciste, le eseguono con il maggior disprezzo possibile.

La situazione attuale del bilinguismo è il risultato del 'Pacchetto', ovvero il provvedimento elaborato dal governo riguardante il governo italiano e austriaco su citato. Prima, per decenni gli abitanti della regione dovevano confrontarsi con I'italiano che spesso gli provocava dei problemi. Bisogna anche ricordare che dopo la seconda guerra mondiale viene interrotta l'italianizzazione forzata: i sudtirolesi ritengono di avere ufficialmente il diritto di usare la propria lingua. Rimane, però, una grande differenza tra la teoria della legge e la pratica della burocrazia italiana: «nessun modulo, nessun formulario, nessun cartello era in tedesco; nessun

2 Termine con cui vengono chiamati gli abitanti germanofoni dell'Alto Adige.

194

Fabrica Litterarum Polono-Italica | 2019, nr 1 (1) 
impiegato statale parlava tedesco; nessun impiegato capiva tedesco» (Melandri 2017: 44). La generalità del problema viene mostrata nel libro a partire dall'esempio della famiglia di Gerda:

Solo Johanna [madre di Gerda] in famiglia sapeva leggere e scrivere in italiano: era l'unica ad aver fatto le scuole fasciste. Hermann aveva frequentato le elementari nell'impero austroungarico, interrompendo gli studi quando gli erano morti i genitori. I figli erano andati alle scuole della Repubblica nata dall'antifascismo, che non aveva restituito il Sudtirolo alla Madre Austria come i suoi abitanti si erano illusi facesse, ma almeno aveva riconosciuto loro il diritto di studiare nella propria lingua. La burocrazia però era ancora tutta in italiano (Melandri 2017: 43).

La situazione è molto complicata e poco gradevole per la società sudtirolese. È una italianizzazione senza appoggio regolare, sottile, ma d'altronde percepibile e penosa.

Un altro momento cruciale della lingua utilizzata come uno strumento di discriminazione, o perlomeno di umiliazione, ha luogo durante il parto di Gerda. La giovane madre viene cacciata da casa da suo padre e trova riparo in un ospizio tenuto dalle suore. La sua situazione è precaria e lei viene stigmatizzata in modo duplice: dalla propria comunità tirolese per essere madre nubile e dalle autorità (qui raffigurate nella persona di una suora infermiera, proveniente dal Sud Italia) per la scarsa padronanza della lingua:

la suora infermiera [...] con disprezzo, disse all'ostetrica:

"Questa manco "spingi" capisce.»

Gerda aspettò che la doglia passasse, poi squadrò la suora e disse:

«lo capiscio.»

La suora infermiera fece una smorfia incredula.

«Kapiscio...!»» ripetè scimmiottando l'accento tedesco di Gerda. Scoppiò a ridere.

«Kapiscio...». II riso le faceva sobbalzare le spalle ossute, non riusciva a fermarsi [...].

"Kapiscio...» continuò a ripetere la suora mentre usciva dalla stanza. Le sue risate risuonarono per tutto il corridoio, finché oltrepassò la porta a vetri che chiudeva il reparto (Melandri 2017: 105-106).

Il brano mostra il disprezzo da parte degli italiani (alcuni) verso i sudtirolesi, una profonda incomprensione della caratteristica della terra dove si trovano e appunto il livello di padronanza della lingua come indicatore per valutare una persona. La suora infermiera non è in grado di comprendere che gli errori linguistici di Gerda,

195

Fabrica Litterarum Polono-Italica | 2019, nr 1 (1) 
in una situazione così particolare come quella del parto, non implichino che la ragazza non sia intelligente.

Non sorprende perciò che, prendendo in considerazione tali esperienze, la gente germanofona si inibisse nel parlare italiano: «non osò chiedergli informazioni: a parlare in italiano si vergognava» (Melandri 2017: 56).

Nel corso degli anni il bilinguismo dei sudtirolesi/altoatesini si è diffuso sempre di più. È un bilinguismo studiato, non innato. Si sente che l'italiano dei sudtirolesi non è un italiano appreso a casa, in modo naturale. L'accento tedesco è sempre ben marcato, non importa quanto bene uno conosca l'italiano. Nella seconda metà degli anni Sessanta, durante i lavori sulle soluzioni pacifiche per stabilizzare le tensioni nella regione, «i [politici] sudtirolesi avevano sorpreso gli interlocutori con il loro italiano forse rigido ma corretto; nessuno dei delegati governativi preposti alla risoluzione della questione altoatesina, invece, aveva reputato necessario imparare una sola parola di tedesco» (Melandri 2017: 199). Le parole citate sono segno di ignoranza da parte dei delegati che non si mostrano capaci di fare un gesto verso i loro interlocutori sudtirolesi e imparare qualcosa della loro lingua - una delle cose più significative per la loro identità. Danno, invece, per scontato che essi, per il solo fatto di trovarsi in Italia, parlino italiano.

Trattando della lingua bisogna fare anche un'altra osservazione. Non è preso in considerazione solo quale lingua si parlasse e come ciò fosse visto dall'esterno, ma anche il mondo in cui tali lingue fossero espresse. In effetti, sia il tedesco che l'italiano parlati nella regione sono molto particolari. Delle influenze italiane nel tedesco si è già accennato nel paragrafo riguardante le bestemmie. Come viene sottolineato nei vari testi specialistici, anche l'italiano della provincia di Bolzano è caratterizzato per via dagli influssi del tedesco (Cavagnoli 2000: 374). Tale questione è segnalata anche nel romanzo. L'unico protagonista altoatesino italofono parla in maniera rappresentativa per il suo gruppo:

«Finita Pasquetta, se vuoi, vengo dentro» [...]. Un altoatesino, pur se di sangue veneto-calabrese come lui, traduce in italiano molte espressioni del nostro dialetto tedesco. Si va dentro, inni, quando si va nelle valli che scorrono aussi, fuori, verso la pianura e il vasto mondo (Melandri 2017: 21).

L'autrice non è però coerente. Da una parte il lettore apprende i problemi linguistici dalla gente, dall'altra non lo percepisce nel linguaggio della narrazione, in maggior parte dei casi i protagonisti parlando non usano nessuna inflessione dialettale. Si può presupporre che la scelta sia basata sulla volontà dell'autrice di farsi capire bene dai lettori e di non far perdere il significato delle importanti tematiche del libro, solo a causa dell'incomprensione linguistica dettata dal lin- 
guaggio dei personaggi. Allo stesso tempo tale decisione può essere vista come una limitazione, un impoverimento del romanzo.

\section{L'incomprensione delle caratteristiche locali da parte degli italiani di fuori provincia}

Nel romanzo vengono descritti così tanti tipi e sfumature della mancanza di presa di coscienza nei rapporti reciproci che sembra opportuno e lecito dedicare all'argomento più spazio. In tutto il romanzo si trovano tantissime figure di italiani (sia i semplici immigrati che i politici o i delegati di governo) che non sanno cosa sia l'Alto Adige, perché vi siano in vigore le leggi speciali e perché gli abitanti vi parlino altre lingue oltre all'italiano.

Gli emigranti del Sud vennero in Alto Adige cercando migliori condizioni di vita, non sapendo che cosa aspettasse loro. Non erano preparati a trovarsi in un posto così culturalmente diverso dal loro mondo:

Agli immigrati che venivano dall'Italia del Sud nessuno aveva spiegato in che posto stessero andando. A nessuno [...] era mai venuto in mente di informarli che stavano per trasferirsi tra gente che parlava tedesco e non mangiava gli spaghetti [...]. Era sempre Italia, no? Questo era tutto ciò che a un migrante serviva sapere (Melandri 2017: 44-45).

Si può solo immaginare lo shock che dovevano vivere gli immigrati all'arrivo nella regione di destinazione. Anche gli emigranti in Germania ingenuamente provavano invidia verso colui che rimaneva al territorio italiano invece di varcare la confine: «sempre lo invidiavano, quando lo vedevano scendere al di qua del Brennero. Non sapevano che sì, quella era ancora Italia, ma per modo di dire» (Melandri 2017: 292). Chiamando I'Alto Adige "l'Italia per modo di dire», l'autrice esprime il suo parere sulla lontananza culturale tra la provincia di Bolzano e il resto del Paese.

L'altro aspetto interessante presentato nel libro concerne l'inquadramento da parte degli italiani della regione nel proprio modo di pensare. Si autorizzano da soli a decidere chi sono gli abitanti della piccola provincia montanara: «Cotoletta alla milanese, viner snizzel [ortografia originale], che differenza vi fa? Ormai tanto siete italiani!» (Melandri 2017: 199). È una battuta pronunciata dai delegati del governo italiano durante la cena con i politici sudtirolesi: essa mostra piena ignoranza e incomprensione della specifica regionale. In più, presenta le autorità italiane come coloro che vogliono risolvere in modo autorevole, come dovrebbe identificarsi la gente di cui non sanno niente. 
Nella parte svoltasi contemporaneamente, a casa del protagonista telefonano i bambini «dal forte accento romano» (Melandri 2017: 19) che si sentono legittimati a decidere anche loro dove dovrebbero vivere i sudtirolesi, cosa che mostrano gridando: «tornatevene in Germania!» (Melandri 2017: 20). La scena fa vedere che anche nell'Italia odierna manca l'educazione su cosa sia l'Alto Adige e perché si trovi in Italia. Fa capire anche che gli stereotipi sono sempre vivi e la popolazione altoatesina viene sempre vista come un'aggiunta straniera alla terra italiana. La telefonata dei bambini meridionali che a prima vista sembra solo una cosa di poca rilevanza mostra che il problema dell'incomprensione da parte italiana è sempre vivo.

Bisogna però ammettere che nel romanzo si trova anche un altro schema di comportamento degli italiani nei confronti dell'Alto Adige. Viene attribuito soprattutto ai personaggi più recenti. Gli italiani chiedono ai sudtirolesi stessi chi si sentano: "la moglie mi guarda e, a tradimento: "Scusi se glielo chiedo ma... lei cosa si sente, più tedesca o più italiana?"»(Melandri 2017: 260). Già dall'inizio del romanzo viene spesso sottolineata l'insensatezza di tale domanda. Ma per conoscere le ragioni per cui la protagonista la ritiene così impropria il lettore deve aspettare quasi fino alle ultime pagine del libro. Intanto il quesito ritorna più volte.

A prima vista la domanda sembra garbata e cortese. Gli italiani sono capaci di ammettere i propri limiti di istruzione per quanto riguarda la specifica locale. Che cosa allora infastidisce la protagonista del romanzo? Si tratta di un'esclusione nascosta nella domanda: la possibilità di scelta molto limitata. Secondo l'opinione sottintesa di chi chiede si può solo essere o italiano o tedesco. Non ci sono identità più vincolate, non c'è spazio per la coesistenza di più influssi né per il legame con la terra. La questione rispecchia lo stesso problema che sorge parlando del censimento obbligatorio per gli abitanti della provincia.

\section{Gli stereotipi reciproci}

L'incomprensione tra i gruppi linguistici ha generato gli stereotipi reciproci italo-tirolesi. Essi permettono a ogni gruppo di rafforzare la propria diversità e di rinchiudersi in essa.

È vero che nel libro si trovano di più segni di pregiudizi e incomprensioni da parte degli italiani, ma ogni tanto vi sono pure quelli tirolesi.

Cominciando da quelli italiani, li si può dividere tra negativi e positivi. I negativi sono legati, soprattutto, alla lingua tedesca parlata dalla gente e alle associazioni che essa provoca negli italiani. Considerando la storia regionale, non sorprende che l'altro filo tematico presente negli stereotipi sia il terrorismo. 
Nonostante non furono i tirolesi a scegliere l'uniforme né il fronte su cui lottavano, una delle immagini più comuni tra gli italiani è quella del sudtirolese-nazista. Quando compare nella televisione italiana il già citato Silvius Magnago, uno dei più importanti politici sudtirolesi al quale si deve il Pacchetto, «il mezzo busto Rai l'aveva presentato così: Silvius Magnago, leader del Südtiroler Volkspartei [Partito Popolare Sudtirolese], ex ufficiale della Wehrmacht» (Melandri 2017: 222). Non aiutano le spiegazioni e gli argomenti: uno stereotipo legato al nome dell'esercito nazista è più forte e discredita il più celebre politico sudtirolese inseguendo la pacificazione della regione.

Gli altoatesini vengono visti come nazisti per il solo fatto di parlare tedesco e combattere nell'esercito tedesco:

Magnago sapeva bene che lo spigoloso accento tedesco con cui parlava, peraltro a perfezione, la lingua di Dante, e il fatto che avesse svolto servizio in guerra nella Wehrmacht, creavano nei suoi interlocutori un'immediata associazione con il nazismo (Melandri 2017: 200).

Ed è un'opinione impossibile da cambiare: non bastava avere una buona padronanza linguistica e allo stesso tempo condannare i terroristi locali, né dedicare la maggior parte della vita a trovare una coesistenza pacifica tra i due gruppi. Dal romanzo di Melandri viene fuori l'immagine degli italiani per i quali gli argomenti contano meno che i pregiudizi.

A proposito dello stereotipo del terrorismo altoatesino, una delle protagoniste che ha più contatto con la gente al di fuori della provincia esprime la seguente osservazione: "fino a qualche anno fa, quando dicevi che eri altoatesino di lingua tedesca ti davano del terrorista. O come minimo ti chiedevano: ma perché voi li odiate così tanto, gli italiani?» (Melandri 2017: 260).

Accanto agli stereotipi e alle opinioni negative, ci sono anche quelle positive, tra cui l'attribuzione dell'ordine e della ricchezza ai sudtirolesi. Un soldato in servizio in Alto Adige ammette: "Una cosa sugli altoatesini, però, non si poteva negare: quella era gente precisa, pulita, che dava all'ordine un sommo valore» (Melandri 2017: 168).

Invece un siciliano, incontrato da Eva nel treno, si riferisce alle autonomie locali che sono in vigore sia nell'Alto Adige che in Sicilia. Come spesso in Italia gli abitanti del Sud, da una parte fortemente legati anche loro alle proprie terre, vivono con una profonda convinzione che al Nord si viva meglio:

Voi l'autonomia regionale ce l'avete davvero! Mica come noi in Sicilia, che siamo autonomi dallo Stato italiano, ma sudditi della mafia. Ricominciassi la carriera, mi trasferirei al Nord e farei crescere i figli lì. Senza tutti quei raccomandati (Melandri 2017: 260). 
Anche se le ultime due citazioni trasmettono delle opinioni molto positive, in realtà non sono niente più che altri stereotipi, cioè modelli generici, convenzionali e semplificati (Treccani... 2018).

Come accennato, accanto agli stereotipi degli italiani ci sono anche quelli dei sudtirolesi, che al contrario non sono molto presenti nel romanzo. Uno dei pochi brani che si potrebbe evocare riguarda l'opinione con cui si doveva confrontare un giovane militare italiano: "I Walschen ${ }^{3}$ sono disonesti, e con prepotenza da militari ne approfittano per non pagare» (Melandri 2017: 244). Evidentemente però la simpatia dell'autrice sembra andare verso gli autoctoni, perché la citata opinione viene subito motivata dalle truffe dei militari italiani che non pagavano per il cibo comprato dai contadini locali.

Per concludere la parte dedicata agli stereotipi e ai pregiudizi reciproci, vale la pena citare due frasi emblematiche che scambiano tra sé due altoatesini: una di lingua tedesca e l’altro di lingua italiana:

"La maggior parte degli altoatesini di lingua italiana pensano che voi sudtirolesi di lingua tedesca siete tutti nazisti».

"La maggior parte dei sudtirolesi di lingua tedesca pensano che voi altoatesini di lingua italiana siete tutti fascisti» (Melandri 2017: 238).

L'umorismo delle espressioni consiste nel mostrare in modo esagerato il meccanismo di funzionamento dello stereotipo. Tutte e due sono opinioni comuni, generiche e superficiali. Si basano sull'antagonismo tra le popolazioni, hanno radici storiche e, soprattutto, i due gruppi si attribuiscono a vicenda gli stessi difetti e vizi e rimandano uno all'altro le stesse colpe.

\section{La separazione dei gruppi linguistici e il censimento etnico}

Dopo l'approvazione, ottenuta da Magnago, del Pacchetto, le popolazioni locali - italiana e tedesca - vengono del tutto separate. Era infatti, questo, uno degli obiettivi principali del politico:

Era fondamentale che le comunità etniche dell'Alto Adige fossero censite, quantificate, e divise chiaramente l'una dall'altra: le scuole e gli istituti culturali e linguistici, soprattutto, perché solo separando da quella italiana la cultura e la lingua sudtirolese la si poteva proteggere efficacemente (Melandri 2017: 304).

3 Un modo spregiativo di chiamare gli italiani nell'Alto Adige.

200

Fabrica Litterarum Polono-Italica | 2019, nr 1 (1) 
Secondo il romanzo Magnago riteneva che le popolazioni dovevano essere divise. Magari tale determinazione si potrebbe giustificare con le esperienze del tempo del fascismo. Rimane però aperta la domanda se siano attuali anche nei tempi della globalizzazione. E infatti tale quesito ritornerà nella narrazione di Melandri.

Prima intanto occorre soffermarci sulle opinioni attribuite dall'autrice al famoso politico altoatesino. Secondo il Magnago fittizio la divisione è necessaria per impedire il mescolarsi delle culture: un processo che provocherebbe il rischio di diminuzione della specifica culturale, linguistica e perfino etnica del Sudtirolo:

Mescolanze e confusioni tra le comunità avrebbero portato, una volta di più, alla deflagrazione, al caos [...]: i Mischehen tra italiani e tedeschi erano l'inizio della fine per il Sudtirolo (Melandri 2017: 305).

E in un altro passaggio del romanzo:

"Nicht Knödel mit Spaghetti mischen», canederli e spaghetti non vanno mescolati. Scuole, biblioteche, assessorati, centri culturali: tutto doveva essere separato (Melandri 2017: 262).

Ed infatti così succede, come è stato già segnalato prima. Lo attesta anche una delle protagoniste di Melandri nella parte del libro ambientata contemporaneamente:

Ora sono sul trenino locale che mi porta verso Fortezza/Franzensfeste. Appeso in alto, in fondo dal vagone, c'è un poster del Deutsches Kultur- und Familienamt, l'assessorato alla famiglia e alla cultura per la popolazione in lingua tedesca rigorosamente distinto è separato dal suo omologo per gli italiani (Melandri 2017: 58).

Per facilitare l'approvazione della separazione è stato introdotto lo strumento legale chiamato "censimento etnico», che serve a stabilire a quale etnia appartenga un individuo. Il problema più rilevante del censimento è l'impossibilità di identificarsi con più di un'etnia. Per la legge non esiste l'identità multipla né mista. Inoltre la scelta è vincolante ed ha un grandissimo impatto sulla vita quotidiana.

Ogni abitante adulto dell'Alto Adige doveva dichiararsi tedesco, ladino o italiano. Chi si rifiutava di compilare la dichiarazione non avrebbe potuto insegnare, chiedere contributi statali, lavorare nel pubblico impiego. Soprattutto definirsi multietnici non si poteva (Melandri 2017: 262).

201

Fabrica Litterarum Polono-Italica | 2019, nr 1 (1) 
In tutto il libro si trovano tantissimi riferimenti critici nei confronti del censimento. L'autrice non nasconde la sua opinione scettica. Introduce nel libro figure di tanti protagonisti che non si inquadrano nella suddivisione facile voluta dal censimento. L'autrice mostra ai lettori due figure-simbolo per farci capire gli stretti limiti di questa soluzione legale.

Il primo è il caso del Signor Song. È un emigrante dal Sud-Est della Cina che abita attualmente con la sua famiglia in Alto Adige, dopo aver vissuto in diverse città italiane. I figli del Signor Song sono nati in Italia. Abitando nel Tirolo del Sud, la famiglia dei migranti cinesi fu costretta a censirsi:

[...] al censimento del 2001, gli fu chiesto di mettere una croce su una di tre caselle: italiano, tedesco o ladino. Nessun'altra possibilità era ammessa, solo queste tre sono le etnie riconosciute in Alto Adige. Per partecipare dei benefici della Regione a statuto speciale era necessario compilare e firmare la dichiarazione d'appartenenza al gruppo linguistico. L'intestazione del formulario, in tedesco, diceva: Sprachgruppenzugehörigkeitserklärung (Melandri 2017: 22).

Il Signor Song sceglie l'italiano per lo spavento che prova nel vedere la parola tedesca con troppe sillabe.

Un personaggio del genere è introdotto nel libro per mostrare in modo evidente le lacune del sistema di censimento. Anche un migrante per poter usufruire dei servizi nella provincia si deve dichiarare appartenente a uno dei gruppi.

L'altra figura, invece, è quella di un barista incontrato casualmente:

Il barista è gentile e parla bene sia l'italiano che il tedesco, con marcato accento bolzanino, ma ha viso, pelle e gesti maghrebini. Chissà al censimento che casella ha barrato sul modulo (Melandri 2017: 89-90).

Il personaggio è allo stesso tempo sia un locale che un forestiero. Locale per le competenze linguistiche già acquisite, caratteristiche per i sudtirolesi, seppure con un accento locale (bolzanino), ma anche forestiero per l'aspetto fisico («viso maghrebino»). Come tutti gli altri abitanti della regione, per avere i pieni diritti (e pure per poter lavorare in Alto Adige) doveva scegliere una etnia tra quella tedesca, quella italiana e quella ladina.

Dalla descrizione il lettore capisce che il barista, come il cinese, non entra facilmente in queste categorie. Qualsiasi sia la sua scelta, sarà sempre una decisione che non rispetta la sua vera origine. È un altro simbolo di insufficienza e inefficienza del sistema. Allo stesso tempo è anche un segno di quanto le classificazioni etniche non siano adatte per il mondo di oggi. Magari prima dei tempi della globalizzazio-

202

Fabrica Litterarum Polono-Italica | 2019, nr 1 (1) 
ne e delle grandi migrazioni tale soluzione avrebbe potuto funzionare - sembra voler dire l'autrice - ma ora, con i cambi radicali del mondo il vecchio modello non è più adatto.

\section{Conclusioni}

Nel presente saggio sono state enunciate e commentate le particolarità che sono le principali responsabili della specifica locale dell'Alto Adige che vengono trasmesse anche nella narrazione. Sono stati messi in evidenza temi come: la lingua e il suo ruolo, l'immagine reciproca - spesso stereotipata - delle due popolazioni e i loro rapporti, le questioni della divisione politica e del censimento etnico. È stata analizzata la presenza di motivi sopra menzionati nel romanzo Eva dorme di Francesca Melandri e il ruolo che essi rappresentano.

L'autrice italiana prova a mostrare al lettore la complicità della realtà altoatesina e le differenze che da essa derivano. È molto abile nell'avvicinare le vicende storiche e politiche mentre, oserei dire, nel romanzo mancano i racconti della vita comune dei due gruppi. Vi si trovano le descrizioni di rappresaglia da parte degli italiani, i rapporti con i turisti, l'atteggiamento e l'incoscienza dei migranti; sono assenti invece le informazioni sugli altoatesini di lingua italiana che condividevano la vita quotidiana con gli altoatesini di etnia germanica. II fatto è ancora più interessante se si tiene presente che tre tra i protagonisti germanofoni avevano compagni italiani. In tutti e tre i casi non sono nemmeno accennati i problemi o le differenze culturali.

È da considerare il punto più debole del libro: nella narrazione appaiono le espressioni tedesche, viene sottolineata la differenza tra le due mentalità, sono rispecchiati i diversi modi di pensare e agire dei protagonisti, c'è un vastissimo elenco dei personaggi di cui ognuno rappresenta uno specifico atteggiamento verso la propria terra e identità, ma comunque la relazione amorosa interetnica è mostrata come ideale, privo di problemi o fraintendimenti. Non si trova nel libro nessuna incomprensione tra i due protagonisti, basata sui problemi linguistici (nemmeno siamo informati in che lingua interagiscono), non ci sono le problematiche differenze nelle abitudini o tradizioni. Sembra che il fatto di provenire non solo da due lati opposti del Paese, ma anche da due lingue e tradizioni ben diverse non influisca affatto sui protagonisti.

Tale mancanza è sorprendente considerando la cura dell'autrice nel corso del romanzo nel cogliere la regione in un'ottica più ampia e polifonica.

203

Fabrica Litterarum Polono-Italica | 2019, nr 1 (1) 


\section{Bibliografia}

BLANCO Luigi (2006): Storia e identità culturale in una regione di confine: il Trentino-Alto Adige/Südtirol. "Scienza e Politica", Vol. 18, n. 34. Online: https://scienzae politica. unibo.it/article/view/2855/2252 [accesso: 20.03.2017].

Cavagnol Stefania (2000): L'italiano degli italofoni in Alto Adige. In: Heimat. Identità regionali nel processo storico. A cura di A. Pasinato. Roma, pp. 367-377.

Garzanti Linguistica. Online: https://www.garzantilinguistica.it/ricerca/?q=bestem mia [accesso: 16.08.2018].

GolEMo Karolina (2013): Trydent-Górna Adyga - alpejski świat na styku kultury łacińskiej i germańskiej. In: Włochy wielokulturowe. Regionalizmy, mniejszości, migracje. Red. K. Golemo. Kraków, pp. 99-121.

Hempel Andreas Gottlieb (2008): Culturonda. Alto Adige-Sudtirol. Itinerari tra storia, cultura e tradizione. Vienna-Bolzano.

KLEINERT Susanne (2012): I/ problema identitario nell'Alto Adige: discorso politico e letteratura a confronto (Joseph Zoderer, Francesca Melandri). Online: https://publi kationen.sulb.uni-saarland.de/bitstream/20.500.11880/23671/1/Kleinert_AltoAdige. pdf [accesso: 30.04.2018].

MARCANTONI Mauro, POSTAL Giorgio (2014): Südtirol. Storia di una guerra rimossa (1956-1967). Roma 2014.

MELANDRI Francesca (2017): Eva dorme. Milano.

STERNALSKI Piotr (2013): Monokulturowość Włoch w okresie faszyzmu. Italianizacja mniejszości narodowych i ustawodawstwa rasowe. In: Włochy wielokulturowe. Regionalizmy, mniejszości, migracje. Red. K. Golemo. Kraków, pp. 51-72.

Treccani. Vocabolario. Online: http://www.treccani.it/vocabolario/stereotipo/ [accesso: 20.08.2018].

VASSALLI Sebastiano (2015): II confine. I cento anni del Sudtirolo in Italia. Milano.

\section{Abstrakt \\ Osobliwości południowotyrolskie we współczesnej prozie włoskiej: na przykładzie powieści Eva dorme Franceski Melandri}

Artykuł porusza zagadnienie obecności Południowego Tyrolu we współczesnej literaturze włoskojęzycznej. Autorka stara się pokazać, w jaki sposób poszczególne cechy charakterystyczne dla alpejskiej prowincji zostały zrealizowane w powieści Eva dorme Franceski Melandri (2011). Wśród omówionych problemów znalazły się: kwestia wielojęzyczności regionu, brak zrozumienia między poszczególnymi grupami etnicznymi oraz relacje z przybyszami spoza prowincji, stereotypy i wzajemne uprzedzenia oraz prawnie narzucone rozdzielenie grupy włosko- i niemieckojęzycznej. Powieść, ze względu na

204

Fabrica Litterarum Polono-Italica | 2019, nr 1 (1) 
poruszaną tematykę, jest ciekawą i oryginalną pozycją na włoskim rynku wydawniczym, można w niej jednak dostrzec zachowawczy sposób przedstawiania lokalnej tożsamości regionalnej oraz aspiracje dydaktyczne, służące przybliżeniu włoskiemu czytelnikowi mało znanej części kraju.

Słowa kluczowe: Południowy Tyrol, Górna Adyga, współczesna literatura włoska, tożsamość regionalna, Francesca Melandri 